\title{
Ulteriori osservazioni sul significato di parbār (1CR 26,18b) alla luce di alcune versioni arabe
}

Giancarlo TOLONI

Seminario Vescovile Diocesano, Brescia

L'esame di $1 \mathrm{Cr} 26,18$ ha consentito di comprendere che il sostantivo parbār, che ricorre alla fine del versetto, è di difficile traduzione, tanto che nella maggior parte delle versioni moderne è solo traslitterato ${ }^{1}$. Esso designa uno spazio aperto nell'area occidentale del tempio ${ }^{2}$, distinto dal resto dell'edificio sacro e, probabilmente, recinto da colonne, la cui funzione pare quella di raccogliere le offerte di purificazione dei sacerdoti e di separarle, quindi, da quelle del popolo ${ }^{3}$.

\section{LO STATUS QUAESTIONIS}

Nel passo si riporta una delle disposizioni di David relative all'organizzazione dei portieri del tempio, specificando che all'in-

1 G. TolonI, «A proposito di *parwār / parbār», AION 56 (1996) pp. 441457; per le versioni moderne cf. ibid n. 20. Le sigle di riviste e collezioni citate nel corso di questo studio sono conformi a R. NORTH, Elenchus of Biblica (Roma 1996) vol. IX/1993 pp. 7a-39b. Le traduzioni dei passi esaminati, dove non si diano indicazioni specifiche, sono da attribuirsi all'a.

2 In F. ZoRell, Lexicon Hebraicum Veteris Testamenti (Romae 1951-1984; rist. 1989) p. 665b, s.v. parbār; L. KoeHLer, W. BAUMGARTNer, J. J. STAMm, Hebräisches und aramäisches Lexikon zum Alten Testament $\left(3 .^{\mathrm{a}}\right.$ ed., Leiden 1983) vol. III pp. 905b-906a, s.v.

3 D. Runnals, «The parwār: A Place of Ritual Separation?», VT 41 (1991) pp. 324-331, spec. pp. 324, 328-329. 
gresso situato sul lato ovest furono disposti sei portieri, ripar-

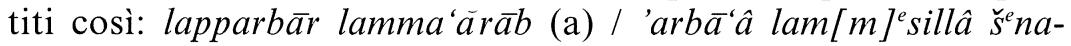
yim lapparbār (b) ${ }^{4}$. Anzitutto si noti che la prima occorrenza del termine in esame deve espungersi, perché probabile dittografia 5: lo scriba riportò anche all'inizio del versetto la locuzione lapparbār, che figura alla fine del medesimo e che potrebbe essere trasposta opportunamente alla fine del v. 17. Quindi il passo va tradotto così: «a occidente / [ce n'erano] quattro per la strada e due per il Parbar». Qui parbār (v. 18b) diventa, di fatto, sinonimo di *parwār, che è attestato al pl. parwārîm unicamente in $2 R e 23,11$ e che costituisce una sua variante grafica e fonetica ${ }^{6}$.

Il confronto delle versioni antiche ha confermato quest'assunto mediante tre diverse modalità di traduzione: il calco linguistico, del Targum, dove parbār è reso $k^{e} l a p p e ̂$ bārā' 'all'esterno' 7; la semplice traslitterazione, della $\mathrm{P}^{e}{ }_{\mathrm{S}} \mathrm{ittat}$ ' (le $\bar{p}$ arbar) 8; la

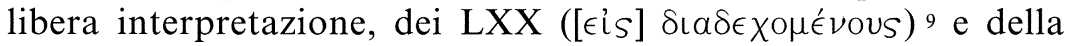
Vulgata (per cellulas) ${ }^{10}$. Fra queste versioni antiche non è stato

4 Biblia Hebraica Stuttgartensia ... (= BHS), ediderunt K. Elliger et W. RUDOLPH (Stuttgart 1967-1977; rist. 1984) p. 1506.

5 BHS p. 1506, apparato critico, n. a al v. 18.

6 Sul significato di questo vocabolo cf. G. TolonI, ""Per non entrare nel tempio" (2Re 23,11aß). Storia dell'interpretazione di un sintagma», EstBíb 55 (1997) pp. 143-169, spec. pp. 152, 159.

7 R. Le Déaut, J. Robert, Targum des Chroniques (AnBib 51; Rome 1971) vol. II: Texte et glossaire p. 71.

8 Biblia Sacra juxta Versionem Simplicem quae dicitur Pschitta (Beryti 1951) vol. I p. 568a

9 Septuaginta, Id est Vetus Testamentum graece iuxta LXX interpretes edidit A. Rahlfs ( $9^{a}$ ed., Stuttgart 1971; rist. 1984) vol. I p. 804. Cf. anche The Old Testament in Greek according to the Text of Codex Vaticanus, Supplemented from Other Uncial Manuscripts, with a Critical Apparatus Containing the Variants of the Chief Ancient Authorities for the Text of the Septuagint, eds. A. E. Brooke, N. McLean, H. St. J. Thackeray (Cambridge 1932) vol. II/3: I-II Chronicles pp. 464-465.

10 Biblia Sacra iuxta Latinam Vulgatam versionem ad codicum fidem ... cura et studio Monachorum Abbatiae Pontificiae S. Hieronymi in urbe ordinis S. Benedicti edita (Romae 1948) vol. VII: Liber Verborum dierum ex interpretatione Sancti Hieronymi ... p. 156b. Cfr. anche Biblia Sacra iuxta Vulgatam versionem ... recensuit et brevi apparatu instruxit R. WEBER, Editio tertia emendata quam paravit B. Fischer cum sociis H. I. Frede, I. Gribomont, H. F. D. Sparks, W. Thiele ( $3^{a}$ ed., Stuttgart 1983: editio minor in un volume solo) p. $581 \mathrm{a}$. 
possibile esaminare anche quella araba riportata nella Poliglotta di Londra ${ }^{11}$, perché l'intera pericope di $1 \mathrm{Cr} 26,12-27,34$ non si è conservata in questa traduzione ${ }^{12}$. Del resto la storia delle versioni arabe delle Cronache è complessa ${ }^{13}$. L. Randellini 14 ricorda che quella antica è strettamente affine alla siriaca, tanto che talora è possibile correggere quest'ultima con quella araba. In alcuni casi il traduttore arabo delle Cronache rivela di non aver capito il testo della $\mathrm{P}^{e}$ šițtā', e se ne discosta; in altri riprende intere parafrasi siriache, tentando di sintetizzarle. Sebbene, con Randellini, si debba ammettere che la traduzione antica, che è tardiva quanto a datazione, sia di scarsa utilità per la ricostruzione dell'originale -se si eccettua $2 \mathrm{Cr}$ 10,2-, tuttavia è assodato che spesso il confronto delle versioni arabe si rivela significativo per la storia dell'interpretazione dell' e che talora può orientare alla soluzione di qualche problema esegetico ${ }^{15}$.

Appare di una certa importanza anche l'esame delle traduzioni arabe moderne, specialmente per il passo che qui interes$\mathrm{sa}$, che è attestato solo in tali versioni. In questo studio ci si occuperà di due edizioni: quella di Beiruth 16 e quella di Cam-

11 Biblia Sacra Polyglotta ..., edidit B. Waltonus (Londini: Th. Roycroft, 1655) vol. II, sez. 1a, p. 702, parte II, col. $1^{\text {a }}$.

12 Nella Poliglotta è attestata la medesima lacuna anche nel testo della versione siriaca (cf. TOLONI «*Parwār» n. 37 e testo corrispondente); pertanto, per l'esame della $\mathrm{P}^{\mathrm{e}}$ šittā' si è dovuto ricorrere all'edizione manuale di Beirut, data l'assenza del volume delle Cronache nell'edizione critica di Leida.

13 Notizie più dettagliate si trovano in G. GRAF, Geschichte der christlichen arabischen Literatur (Studi e Testi 118; Città del Vaticano 1944) vol. I: Die Übersetzungen pp. 93-101.

14 L. Randellini, Il libro delle Cronache (La Sacra Bibbia, Volgata latina e traduzione italiana dai testi originali illustrate con note critiche e commentate sotto la direzione di Mons. S. Garofalo, s.n.; Antico Testamento a cura di P. G. Rinaldi; Torino-Roma 1966) p. 8.

15 E. WÜrTweIn, The Text of the Old Testament, An Introduction to the Biblia Hebraica, ed. ingl. E. F. Rhodes ( $2^{\mathrm{a}}$ ed., Grand Rapids, MI, 1995; ed. orig. Der Text des Alten Testaments [5 $5^{\mathrm{a}}$ ed., Stuttgart 1988]) p. 104.

16 'Al-kitâbu 'l-muqaddasu ... / Arabic Bible (Beirut 1992). Quest'edizione riproduce in un volume unico la primitiva, in arabo classico, edita a Beirut dalla Dar El-Machreq, dei Padri Gesuiti della Missione libanese; essa era suddivisa in tre volumi: il primo (1876) ed il secondo (1880) dedicati all'AT, il terzo (1878) al NT. Ne furono eseguite nuove edizioni nel 1890, 1899, 1911, 1932. La prima edizione anastatica (fotolitografica), in un volume unico, di 
bridge ${ }^{17}$, che sono state effettuate sulla base del TM. Pur non trattandosi di edizioni critiche, esse possono offrire un interessante contributo alla comprensione del significato di parbār, presente nella loro Vorlage, dato che testimoniano uno stadio molto avanzato del processo di dilatazione dell'arco semantico di questo vocabolo.

\section{ANALiSI FILOLOGICA}

Nella prima delle due versioni il versetto è reso così: wamin ğihati 'r-riwâqi ilà 'l-garbi (a) / 'arba'atan fî̀ 'l-mas'adi wa' tnajni fî̀ 'r-riwâqi (b) ${ }^{18}$; nella seconda si legge: min ğihati 'r-rawấqi ilà 'l-garbi (a) / 'arba'atan fî 'l-maș'adi wa' tnajni fî̀ 'r-rawâqi (b) ${ }^{19}$. In entrambe il testo ebraico è tradotto allo stesso modo, con una sola differenza: mentre nella prima il termine in esame è reso con riwâqi, caso obliquo del sostantivo riwâqun 'colonnato, portico' ${ }^{20}$, nella seconda si legge rawâqi, caso obliquo di rawâqun, corradicale del primo, del medesimo significato ${ }^{21}$. I due vocaboli si distinguono solo per la diversa vocale della prima sillaba, rispettivamente kasrah (i) e fathah (a). Inoltre nella seconda traduzione manca la congiunzione coordinante

formato ridotto, è del 1897, edita a Beirut. La Società Biblica Britannica e Forestiera ristampò quest'ultima nel 1987, sempre in un volume unico. Della stessa tipologia è quella qui esaminata, che rappresenta l'ultima ristampa dell'opera originale. Recentemente (1991) è stata diffusa un'altra edizione della stessa Bibbia araba, però vocalizzata solo parzialmente; è in due volumi (AT 2030 págs.; NT 892 págs.), o anche in uno solo, distribuita dalla Società Biblica Britannica e Forestiera (cf. anche GrAF Geschichte pp. 100101).

17 'Al-kitâbu 'l-muqaddasu ... / Arabic Bible (Cambridge 1994). Tale edizione di Cambridge costituisce l'ultima ristampa della Bibbia araba edita dalla Società Biblica Britannica e Forestiera a Londra nel 1854, 1857, 1861, 1872, 1905; a Cambridge 1907 (cf. anche GRAF Geschichte pp. 98-99).

18 'Al-kitâbu ... (Beirut) pp. 714-715.

19 'Al-kitâbu ... (Cambridge) p. 673.

20 Ed. R. TRaInI, Vocabolario arabo-italiano (Pubblicazioni dell'Istituto per l'Oriente 60; Roma 1966; rist. anast. dei tre tomi in un volume unico 1993) vol. I p. 500a, s.v. riwâq; A. de BIBERSTEIN KAZIMIRSKI, Dictionnaire arabefrançais (Paris 1960) vol. I p. 956a, s.v. riwâqun.

21 Il vocabolo rawâqun, però, figura solo in E. W. LANE, Arabic-English Lexicon (London-Edinburgh: Williams-Norgate, 1867; rist. anast. New York 1956) vol. I/3 p. 1191c, s.v. 
$w a$, che nella prima apre il passo. Quindi il versetto può essere reso, in ambedue i casi, nel modo seguente: «(E) dal lato del colonnato ad occidente / [ce n'erano] quattro sulla salita e due nel colonnato». Un'importante osservazione è data dal constatare che in questa versione si introduce un elemento di chiarificazione rispetto alle antiche già studiate; infatti, con il sintagma fî ' $l$-mas'adi 'sulla salita' 22 , si precisa che quella strada conduceva alla città alta, quindi poteva identificarsi, come è stato proposto da alcuni critici circa $I C r 26,16$, con la valle del Tyropaeon ${ }^{23}$. Inoltre altrettanto significativa è la designazione del parbār come 'ar-riwâqun 'il colonnato', spiegazione che si comprende sulla base della seriorità di questa versione. È evidente la preoccupazione del traduttore arabo di rendere chiaramente il senso della prima occorrenza della locuzione ebraica in esame -che egli ha conservato nell'originale, conformemente al TM-, tanto che, rispetto alla seconda, ha integrato qui il sostantivo ğihatun 'lato' ${ }^{24}$, in caso obliquo perché retto dalla preposizione $\min$ ' $\mathrm{da}$ ' ${ }^{25}$, e in st. c. poiché regge 'ar-riwâ$q i$, pure in caso obliquo, con la funzione di spiegare l'esatto punto di tale struttura occidentale del tempio a cui furono preposti quei sorveglianti. Pertanto qui la locuzione ebraica è tradotta similmente a quanto si è visto anche nella Vulgata, dove pure si mantiene la prima occorrenza, rendendola in cellulis 'nelle cellette' ${ }^{26}$, ed integrando, dopo di essa, il sostantivo jani-

22 Traini Vocabolario (Pubblicazioni dell'Istituto per l'Oriente 62; Roma 1969) vol. II pp. 747a-748a, s.v. sa'ida, spec. p. 747b, s.v. mas'ad; invece in BibersteIn KAZIMIRSKI Dictionnaire vol. I pp. 1337b-1338a, s.v. sa'ida, non si annota il sostantivo maș'adun.

23 Cf. per es. S. Virgulin, Libri delle Cronache (Nuovissima versione della Bibbia dai testi originali 10) (2a ed., Roma 1977) p. 220 n. al v. 16; E. L. CuRTis, A. A. Madsen, A Critical and Exegetical Commentary on the Books of Chronicles (ICC s.n.; Edinburgh 1910; rist. 1952) p. 285.

24 Traini Vocabolario (Pubblicazioni dell'Istituto per l'Oriente 69; Roma 1973) vol. III pp. 1659b-1664a, s.v. I wağuha, spec. p. 1660a-b, s.v. 乌̆ihat; BiberSTEIN KAZIMIRSKI Dictionnaire vol. II pp. 1494b-1496a, s.v. wağaha, spec. pp. 1495b-1496a, s.v. ğihatun, ğuhatun.

25 Traini Vocabolario vol. III p. 1434b, s.v.; Biberstein KaZimirski Dictionnaire vol. II p. 1154a, s.v.

26 Ae. Forcellini, Lexicon totius Latinitatis, eds. F. Corradini, I. Perin (Patavii: Typis Seminarii, 1864; rist. anast. 1965) vol. I p. 574b, s.v. cellula; F. CAlonghi, Dizionario latino-italiano, Terza edizione interamente rifusa ed aggiornata del dizionario GEORGES-CALONGHI (Torino 1950; rist. 1972) vol. 
torum 'dei portieri' ${ }^{27}$, in genitivo di specificazione (in cellulis janitorum).

\section{VALUTAZIONE DEI DATI}

A differenza delle versioni antiche considerate, in quelle arabe moderne il sintagma che interessa è reso con una interpretazione libera, pressoché parafrastica. Questo procedimento è l'unico in cui si dà una vera e propria traduzione del passo, tentando perciò di spiegare anche il senso della prima occorrenza del sintagma, che viene conservato all'inizio del versetto; invece, come si è visto, più propriamente essa dovrà espungersi da queste versioni. È evidente che nelle traduzioni arabe $(f \hat{\imath}$ 'r-riwâqi / rawâqi) si utilizza per la seconda locuzione una

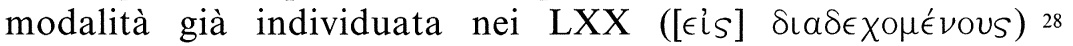
e, soprattutto, nella Vulgata (per cellulas); infatti, con diversi costrutti sintattici e precisazioni lessicali, si dà una lettura contestualizzata di parbār.

In definitiva, come nella Vulgata si aveva una riprova che parbār $(1 C r$ 26,18) deve considerarsi sinonimo di *parwār, dato che al sintagma lapparbār (v. 18b), del TM, corrisponde la locuzione per cellulas 'per le cellette', che riprende esattamente il senso di (bap)parwārîm in $2 \operatorname{Re} 23,11 \mathrm{a}$, così anche nelle traduzioni arabe questa equivalenza semantica è precisata rendendo parbār con riwâqun, sostantivo che significa espressamente 'colonnato', e non solo, genericamente 'recinto (sacro)'. In tal modo si aggiunge anche un'indicazione circa l'ubicazione e la fisionomia di quei locali in prossimità del tempio destinati agli addetti al culto, rimasta implicita nel passo dei $R e$. La conclusione è che, mentre nel TM e nella Vorlage delle versioni antiche parbār designa genericamente, alla luce del contesto, un luogo a parte, all'aperto, nel recinto templare -anche se qual-

I p. 441a, s.v.; Oxford Latin Dictionary, eds. M. Wyllie's, P. G. W. Glare et al. (Oxford 1969) vol. II p. 295c, s.v.; A Latin Dictionary, eds. C. T. LEwIS, S. Short (Oxford: Clarendon, 1879; rist. 1962) p. 310a, s.v.

27 Forcellini Lexicon vol. II p. 955c, s.v. janitor; CAlonghi Dizionario vol. I p. 1286b, s.v. ianitor; Wyllie's - Glare Dictionary (Oxford 1973) vol. IV p. 816c, s.v.; LeWIS - SHORT Dictionary p. 1012b, s.v. janitor.

28 Su quest'espressione cf. G. TOLONI, «La funzione sintattica ed il signifi-

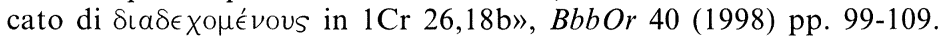


che critico ${ }^{29}$ già lo identifica con l'area delimitata dal portico di colonne posto sul lato occidentale-, in quelle arabe moderne il vocabolo ebraico è reso chiaramente nel significato specifico di 'colonnato'.

Pertanto tali traduzioni arabe danno un'importante conferma che il significato di parbarr, che nell'uso giudaico si precisa evolvendosi a livello semantico ${ }^{30}$, tende a quello di 'colonnato, portico', poiché i vocaboli arabi qui usati hanno esplicitamente questo significato. $\grave{E}$ evidente che $\mathrm{i}$ traduttori di tali versioni moderne applicarono a parbār in $1 C r$ 26,18b un'accezione molto simile a quella suggerita nei mss di Qumran e nel Talmud ${ }^{31}$, dove il vocabolo ebraico non è più inteso semplicemente nel significato di luogo a parte dell'area templare, all'aperto, ma in quello più specifico di luogo a parte, delimitato da una struttura colonnare. Trattandosi di traduzioni tarde ${ }^{32}$, vi si interpreta il testo ebraico alla luce di un processo di sviluppo semantico ormai chiaro. Del resto, se si eccettuano i LXX e la Vulgata, nelle versioni antiche (Targum e $\mathrm{P}^{e}$ šitt $\overline{\mathrm{a}}^{\prime}$ ) il termine era solo ricalcato linguisticamente o traslitterato, segno che il traduttore non ne capiva il senso e ne conservò semplicemente il suono.

29 O. Odelain, R. SÉguineau, Dictionnaire des noms propres de la Bible (3a ed., Paris 1988) p. 287, s.v. parbar; C. COTs, «Netan mélek», in Enciclopedia della Bibbia, eds. A. Rolla, G. Ghiberti, F. Ardusso, G. Marocco (Torino-Leumann 1971; ed. orig. Enciclopedia de la Biblia, eds. A. DíEz MACHO, S. Bartina, J. A. GutiérRez Larraya [Barcelona 1963]) vol. V p. 122; CuRTIS - MADSEN Books of Chronicles p. 285.

$30 \mathrm{Cf}$. W. Jastrow, A Dictionary of the Targumim, the Talmud Babli and Yerushalmi, and the Midrashic Literature (London - New York: Luzac - Putnam, 1886-1903; $2^{\text {a }}$ rist. anast. New York 1950) vol. II p. 1213b, s.v.; G. H. Dalman, Aramäisch-Neuhebräisches Handwörterbuch zu Targum, Talmud und Midrasch ( $3^{\mathrm{a}}$ ed., Göttingen 1938; $2^{\mathrm{a}}$ rist. anast. Hildesheim 1987) p. 345a, s.v.

31 I testi di questa letteratura sono già stati esaminati in TOLONI «*Parwār» pp. 451-455.

32 Graf Geschichte vol. I pp. 98-101. Cf. anche supra nn. 16-17. 


\section{RESUMEN}

En las versiones árabes modernas (ediciones de Beirut y Cambridge) de 1 Cr 26:18, se traduce la palabra parbār por el sustantivo riwâqun / rawâqun 'columnata'. Se trata de una interpretación libre del texto original, similar a la de los LXX y sobre todo a la de la Vulgata. En el curso de la evolución semántica ocurrida en el judaísmo, también se va especificando el significado del término, al igual que el de su variante *parwār. De hecho, en los manuscritos de Qumrán y en el Talmud la palabra parbār se interpreta según el contexto como 'columnata, pórtico', y así lo atestiguan los términos árabes utilizados en las versiones modernas, los cuales tienen expresamente ese mismo significado.

\section{SUMMARY}

In Modern Arabic Versions (editions of Beyruth and Cambridge) of $1 \mathrm{Chr}$ 26:18, the word parbār is translated by the substantiv riwâqun / rawâqun 'colonnade', by a free interpretation of original text, similar to that one of the LXX and, first of all, of the Vulgate. So also this term, as its variant *parwarr, is specified in the course of the semantic evolution happened in Judaism; in fact, in MSS of Qumran and in the Talmud, parbār is contextualized in the sense of 'colonnade, portico', as the Arabic terms used in Modern Versions attest, terms which have expressly such meaning. 